\title{
Introduction to Cyber Manufacturing
}

\author{
Jay Lee, Behrad Bagheri, Chao Jin \\ University of Cincinnati
}

\begin{abstract}
Cyber manufacturing is transformative concept that involves the translation of data from interconnected systems into predictive and prescriptive operations to achieve resilient performance. It intertwines industrial big data and smart analytics to discover and comprehend invisible issues for decision making. With the advent of Internet-of-Things (IoT) and smart, predictive analytics technologies, companies have been building a networked data-rich environment, which calls for a systematic methodology to transform raw data into meaningful and actionable operations. This paper introduces a fundamental framework and architecture for cyber manufacturing systems.
\end{abstract}

\section{Introduction}

Today's manufacturing operations integrate resources in a more complex manner and global scale. Such levels of scale and complexity requires extensive collaboration. Through open innovation, companies can use crowdsourcing to bring in new resources to accelerate the innovation processes [1]. To achieve this, open platforms that consist of a wide spectrum of analytics tools are needed to support design, manufacturing, and services - currently, there are many on-going efforts to accomplish this. For example, GE has announced Predix ${ }^{\mathrm{TM}}$ as a cloud-based service platform to enable industrial-scale analytics for management of asset performance and optimization of operations [2]. National Instruments has introduced Big Analog Data ${ }^{\mathrm{TM}}$, which is a three-tier architecture solution [3], and has ported the Watchdog Agent ${ }^{\mathrm{TM}}$ Toolkit into its LabVIEW development environment to support the rapid creation of smart analytics solutions throughout different big data applications [4], [5]. Germany is supporting Industry 4.0 as a new initiative to transform cyber-physical production systems as next-generation smart manufacturing systems [6].

The number of efforts in this area is exemplary of the need for a multi-scale platform to address the following transformations in tomorrow's manufacturing:

Transformation from machine-based to evidence-based decision making. Traditionally, manufacturing system management heavily depends on experienced personnel. As these personnel, many of which are members of the "baby boom" generation, begin to retire, a great wealth of knowledge, know-how and understanding are lost. Therefore, a smart analytical system is needed to transform experience-based know-how into evidence-based decision making for sustainable operation.

Transformation from solving visible problems to avoiding invisible issues. Manufacturing issues can generally be divided into visible and invisible categories [7]. Through smart analytics of interconnected multidimensional systems, correlations and causal functions can be modeled so that meaningful and actionable operations can be extracted.

Transformation from control-oriented machine learning to data-rich deep learning. Conventional artificial intelligence-based machine learning technologies have been developed primarily for smart 
machine control. However, in a networked, data-rich environment, data conditions are dynamically changing which necessitates greater resilience in modeling of unknown issues. And rather than control, the purpose of cyber manufacturing is to enable users to comprehend the invisible causal relationships and make optimized decisions.

\section{Definition}

Cyber manufacturing is a transformative system that translates data from interconnected system into predictive and prescriptive operations to achieve resilient performance (Figure 1). It intertwines industrial big data and smart analytics to discover and comprehend invisible issues for decision making. In addition, a cyber-physical interface (CPI) plays a key role in cyber security for connected machines (see section 3.2.1).

\section{Cyber Manufacturing System}

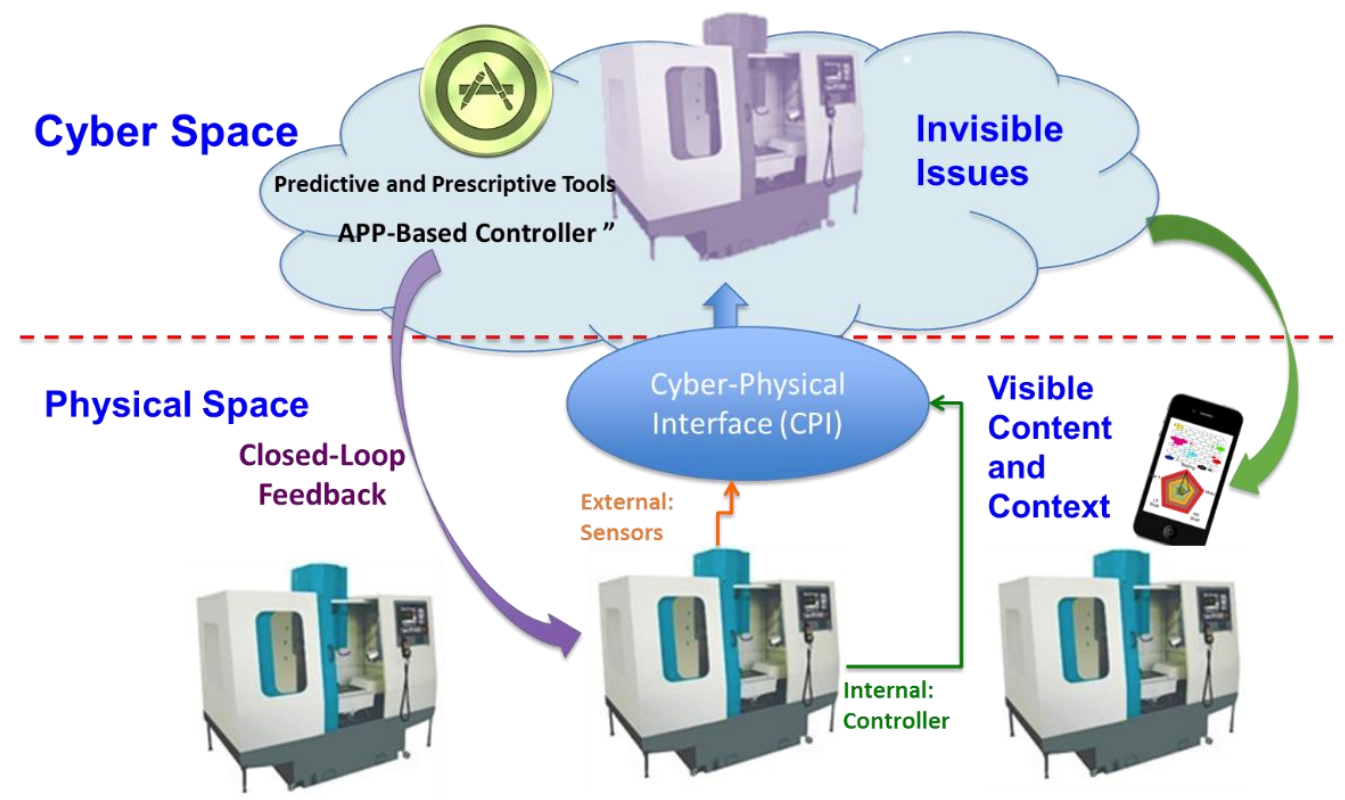

Figure 1 Cyber Manufacturing System

Cyber manufacturing evolved from e-manufacturing, which is a systematic methodology that enables manufacturing operations to successfully integrate with the functional objectives of an enterprise through the use of tether-free (i.e. Internet, wireless, web, etc.) communication and predictive technologies [8]. As indicated in Table 1, both e-manufacturing [8] and cyber manufacturing aim to reduce unexpected downtime and integrate operations with enterprise objectives. However, cyber manufacturing is targeting a system that is much more complex and data-rich, where technologies including smart analytics, distributed systems, control science, and operation management need to be integrated to construct a cyber-physical model [9]. Also, unlike problem-specific solutions in e-manufacturing, cyber manufacturing deploys digital twin technologies to support the life cycle of products. This will enable control systems to compensate or responsible personnel to intervene at the right time on the right assets. Currently, GE is developing Brilliant Manufacturing as a transformational system in the cyber manufacturing environment [10]. 
Table 1. Comparison between E-Manufacturing and Cyber Manufacturing

\begin{tabular}{|c|c|c|}
\hline & E-Manufacturing & Cyber Manufacturing \\
\hline Objective & $\begin{array}{c}\text { Integrate Machine, Production } \\
\text { System, Plant, and Enterprise } \\
\text { with e-Business }\end{array}$ & $\begin{array}{c}\text { Integrated Digital Thread, from } \\
\text { Design, Manufacturing, Services for } \\
\text { Collaborative Innovation }\end{array}$ \\
\hline Data Source & $\begin{array}{c}\text { Sensors \& Controllers \& } \\
\text { Networks }\end{array}$ & All Sources \\
\hline Network Environment & Web-based and Tether-free & Industrial Internet, IoT, Cloud \\
\hline Learning Capability & Programmable & Deep Learning \\
\hline Generality & Application Specific & Generic for Different Assets \\
\hline $\begin{array}{c}\text { Inter-factory Production } \\
\text { Planning }\end{array}$ & Limited & Highly Integrated \\
\hline Self-Configuration & Limited & A Prerequisite \\
\hline Accessibility & Web-based & Mobile \& Cloud-based \\
\hline Scalability & Flexible & Highly Reconfigurable \\
\hline Enabling Technology & $\begin{array}{l}\text { Networked and Remote } \\
\text { Monitoring }\end{array}$ & Cyber-Physical System \\
\hline
\end{tabular}

\section{Challenges}

\subsection{Lack of Standards for Seamless Connectivity}

Compared to existing Internet-enabled industries, manufacturing assets are less connected, and even those assets that are tend to follow customized protocols. In spite of progresses made in CNC machines, which resulted in protocols such as MTConnect [11], the majority of the equipment use different types of sensors, hardware and software, which leads to different data formats and acquisition requirements. Such situations leave end-users faced with challenges to bring seamless connectivity into their manufacturing plants. Currently, there are several initiatives to address standardization issues and to make technologies more open-access. The Digital Manufacturing and Design Innovation Institute (DMDII) has announced an open-source project called Digital Manufacturing Commons (DMC) [12], [13], that enables technology developers to access and interact with data from various vendors. The National Institute of Standards and Technology (NIST) has also endorsed open-source solutions for interoperability of different data sources in a road map of smart manufacturing systems [14].

\subsection{Big Data and Disconnected Analytics}

The massive amount of raw data available from factory floor creates opportunities to add intelligence to the manufacturing process. McKinsey Report indicated that manufacturing has the largest amount of data stored annually [15]. Meanwhile, the volume, velocity, and variety [16] of the generated data have provided industries with a noticeable challenge: how to extract actionable information from this big data. 
To address this issue, machine health analytics need to be effectively integrated with factory operation analytics (Figure 2). In addition, technologies such as Hadoop and Spark have been proposed to provide more powerful computational powers through cloud-based and distributed computing [17]-[21].

\section{Big Data Analytics in Cyber Manufacturing System}

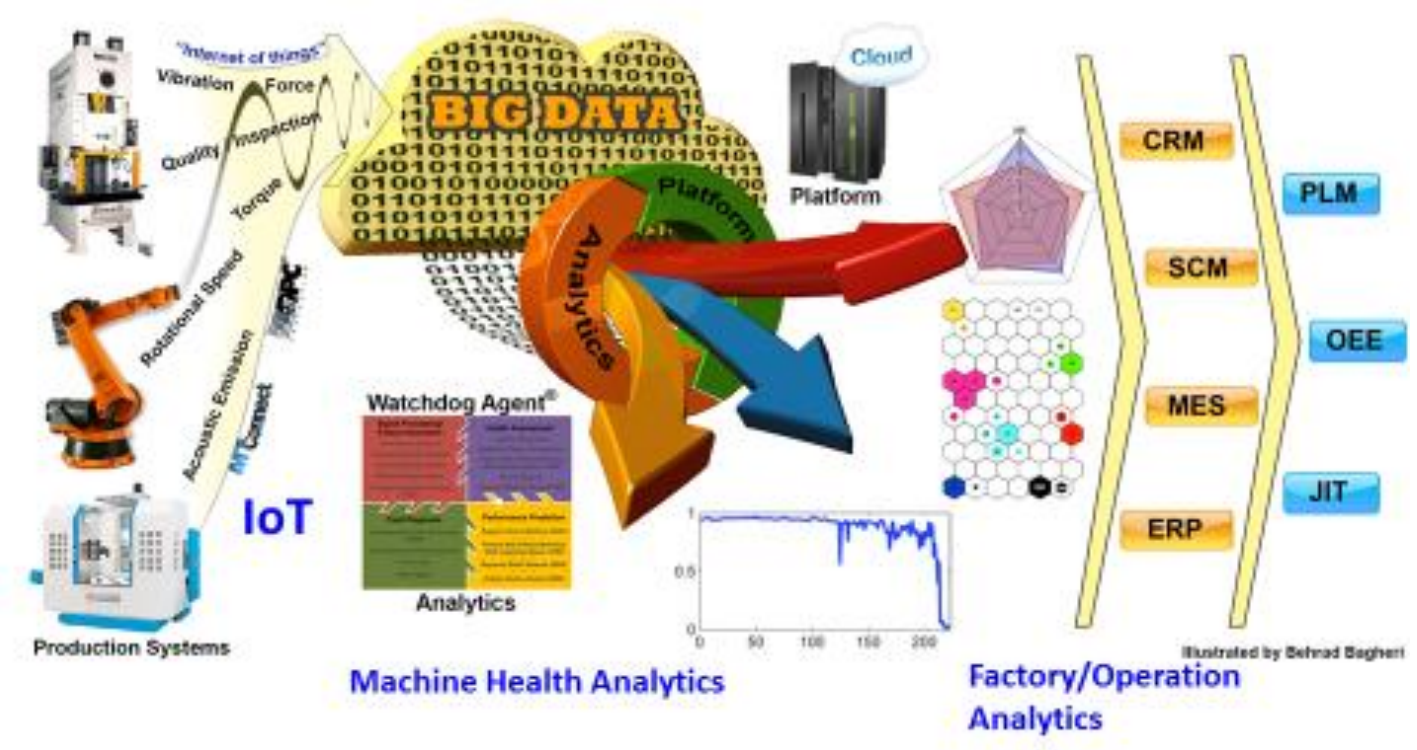

Figure 2 Big Data Analytics in Cyber Manufacturing Systems

\subsubsection{Industrial Cyber Security}

Cyber security is one of the major hurdles in implementing cyber manufacturing as it is critical to have resilient capabilities for connected machines and systems in a cloud environment. One approach is to develop a smart cyber-machine interface (CPI) with a time-machine monitoring function so a virtual testing algorithm can be implemented for any control action as input to the physical machine or system. Cyber attacks can be categorized as general and targeted, the latter of which has increased in recent years. Targeted attacks use customized payloads and have higher impacts on the targeted enterprise [22]. Cyber security requires incorporating intelligence-driven approaches instead of solely depending on tools. For example, Defense in Depth is a traditional cyber security strategy that relies on various tools in each layer to ensure protection against attacks. An intelligence-driven use of Defense in Depth [23] requires continuous monitoring of the network and proactively response to potential attacks. Such strategy results in continuous improvement of the defense-intelligence [22]. In addition, developing strict guidelines, technical standards, and educating personnel to avoid social engineering attacks also play a significant role in supporting cyber-security efforts [19].

\section{Enabling Technologies for Cyber manufacturing \\ 4.1 Internet of Things and Predictive Analytics}

Internet-of-Things (IoT) defines the universe as the digital threads. The on-going rapid pace of implementing IoT has enabled the manufacturing industry to collect data from an increasing number of manufacturing assets. With semiconductor manufacturing at the frontier, machine tools, band saw machines, conveyors and even products are now able to generate diverse sets of data from controllers and 
add-on sensors. The key issues in IoT do not stop at connectivity, but also include how to identify critical assets/components to collect the right data, how to synchronize and bridge different sources of data together, and how to conduct analysis. In fact, predictive analytics is what really translates raw data into actionable information and brings out enormous business values of IoT [24]-[26].

\subsection{Cyber-Physical Systems and Platform}

The core driving technology of cyber manufacturing is cyber-physical systems (CPS). CPS provides seamless integration between computational models and physical components while offering interoperability and resilience [27]. The " $5 \mathrm{C}$ " architecture (Connection, Conversion, Cyber, Cognition, and Configuration) indicates that cyber-physical systems will transfer raw data to actionable operations, assist users to comprehend process information, and, eventually, to add resilience to the system through evidence-based decision making. Cyber Manufacturing Systems consist of two main functional components: (1) advanced interconnected sensing systems that majorly can be realized through IoT; (2) data management and smart analytics capabilities that transforms raw data into predictive and prescriptive operations [9]. Improved operational processes will then reduce lead time and increase productivity, thus making the enterprise more competitive and robust against changing customer needs.

\section{Transformation to Cyber Manufacturing}

As IoT and CPS technologies leap forward, cyber manufacturing realization will be facilitated through standardization between different assets and data hubs, more reliable cyber security, and a general, scalable platform for analytical technologies. Recently, the Center for Intelligent Maintenance Systems has been working on a National Science Foundation-awarded research project on cyber manufacturing [28]. As shown in Figure 3, the enterprises with multi-scale, complex, and networked assets will be able to take advantage of cyber manufacturing to maintain productivity by reducing unexpected downtime, reconfigure production assets based on their health status, and thus inject resilience into manufacturing systems.

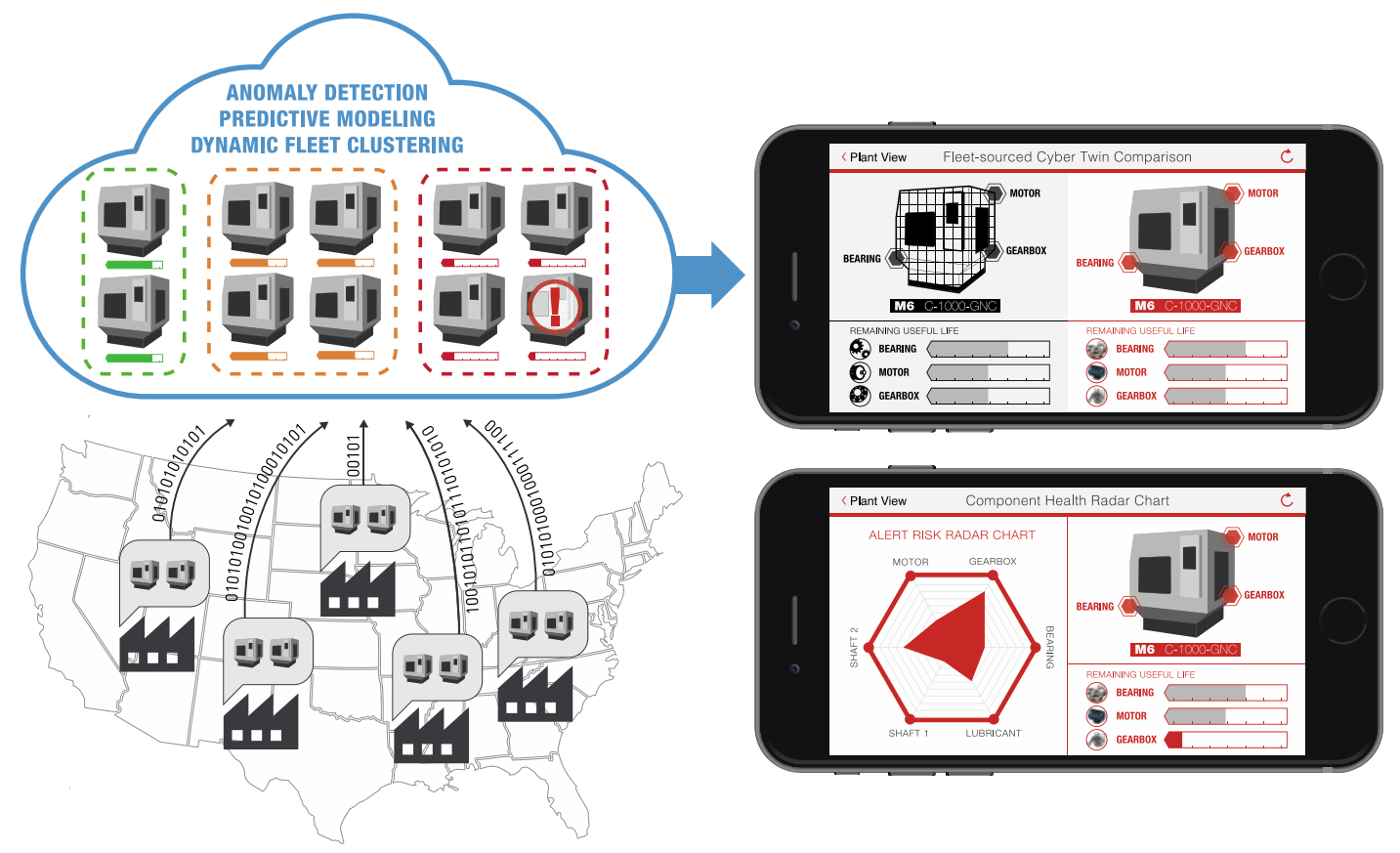

Figure 3 Vision for Cyber-Physical System-enabled Manufacturing 


\section{Conclusions}

This paper introduces the fundamental framework and architecture of cyber manufacturing. With a comparison to e-manufacturing, challenges were identified to be lack of standards, handling of big data, and cyber security. Enabling technologies must overcome these challenges while advancing capabilities of Internet-of-Things, predictive analytics, and cyber physical systems. Transformation from current manufacturing practices to cyber manufacturing requires advances in smart analytics, establishment of standards, and the development of a general platform to enable rapid decision making in a big data environment.

\section{Acknowledgement}

This research is supported by NSF Grant 1550433 under the project call of Dear Colleague Letter: Cybermanufacturing Systems as well as NSF I/UCRC for Intelligent Maintenance Systems.

\section{References}

[1] General Electric, “GE Open Innovation.” [Online]. Available: http://www.ge.com/aboutus/openinnovation.

[2] General Electric, "Predix.” [Online]. Available: https://www.ge.com/digital/predix.

[3] National Instruments, "Big Analog Data ${ }^{\mathrm{TM}}$ Solutions." [Online]. Available: http://www.ni.com/white-paper/14667/en/.

[4] Center for Intelligent Maintenance Systems, "Development of Smart Prognostics Agents (WATCHDOG AGENT®).” [Online]. Available: http://www.imscenter.net/frontpage/Resources/WD.pdf.

[5] National Instruments, "Watchdog Agent ${ }^{\mathrm{TM}}$ Prognostics Toolkit for LabVIEW - IMS Center." [Online]. Available: http://sine.ni.com/nips/cds/view/p/lang/en/nid/210191.

[6] Germany Trade \& Invest, "INDUSTRIE 4.0: SMART MANUFACTURING FOR THE FUTURE."

[7] J. Lee, E. Lapira, B. Bagheri, and H. Kao, "Recent advances and trends in predictive manufacturing systems in big data environment," Manuf. Lett., vol. 1, no. 1, pp. 38-41, Oct. 2013.

[8] J. Lee, "E-manufacturing-fundamental, tools, and transformation," Robot. Comput. Integr. Manuf., vol. 19, no. 6, pp. 501-507, Dec. 2003.

[9] J. Lee, B. Bagheri, and H.-A. Kao, "A Cyber-Physical Systems architecture for Industry 4.0-based manufacturing systems," Manuf. Lett., vol. 3, pp. 18-23, 2015.

[10] "GE Brilliant Manufacturing." [Online]. Available: https://www.ge.com/digital/brilliantmanufacturing.

[11] “MTConnect." [Online]. Available: http://www.mtconnect.org/.

[12] "Digital Manufacturing and Design Innovation Institute (DMDII).”.

[13] The Digital Manufacturing and Design Innovation Institute ( DMDII ), "Digital Manufacturing Commons.".

[14] National Institute of Standards and Technology, "Measurement Science Roadmap for Prognostics and Health management for Smart Manufacturing Systems," Natl. Inst. Stand. Technol., 2015.

[15] McKinsey Global Institute, "Big data: The next frontier for innovation, competition, and productivity," 2011. 
[16] A. McAfee, E. Brynjolfsson, T. H. Davenport, D. J. Patil, and D. Barton, "Big data," Manag. revolution. Harvard Bus Rev, vol. 90, no. 10, pp. 61-67, 2012.

[17] Sas, "Big Data Meets Big Analytics," 2012.

[18] Ronal Berger Strategy Consultants, "Cyber-security: Managing threat scenarios in manufacturing companies (2015)," 2015. .

[19] Rockwell Automation, "Securing Manufacturing Computing and Controller Assets," 2009.

[20] T. Sibalija, "Cyber-security in digital manufacturing: assessment and testing," in 2nd International Conference onf "Modern Methods of Testing and Evaluation in Science," 2015.

[21] IDC and EMC, "The Digital Universe of Opportunities: Rich Data and Increasing Value of the Internet of Things," 2014. .

[22] M. Assante, R. M. Lee, and R. Mahler, “Advanced ICS Cybersecurity: Moving Beyond Defence in-Depth to an Intelligence-Driven Approach,” 2015.

[23] Department of Homeland Security, "Recommended Practice: Improving Industrial Control Systems Cybersecurity with Defense-In-Depth Strategies," 2009.

[24] ABB, "Big Data and decision-making in industrial plants." [Online]. Available: http://new.abb.com/cpm/industry-software/MOM-manufacturing-operations-management/bigdata-analytics-decision-making.

[25] G. E. I. Platforms, “The Rise of Industrial Big Data,” GE Intelligent Platforms, 2012.

[26] J. Lee, Industrial Big Data. Mechanical Industry Press, China, 2015.

[27] N. S. Foundation, "Program Solicitation: Cyber-Physical Systems (CPS)." [Online]. Available: http://www.nsf.gov/pubs/2015/nsf15541/nsf15541.htm.

[28] National Science Foundation, "US NSF - Dear Colleague Letter: Cybermanufacturing Systems (nsf15061)." [Online]. Available: http://www.nsf.gov/pubs/2015/nsf15061/nsf15061.jsp. [Accessed: 11-Apr-2016]. 